\title{
APPORT DE L'INJECTION INTRACAVERNEUSE DE PROSTAGLANDINE E1 LORS DE LA PREMIERE CONSULTATION DE L'IMPUISSANT : à propos de 414 cas
}

\author{
U. CALVET*, B. BALZA**, N. MAILLET****, P. LEANDRI***, G. ROSSIGNOL***, \\ J.R. GAUTIER***, J. CORRAZE* \\ * Institut de Pathologie du Comportement, Clinique Saint Jean, TOULOUSE - ** Pharmacien des Hôpitaux, CARCASSONNE \\ *** Service Urologie, Clinique Saint Jean, TOULOUSE - **** Psychiatre des Hôpitaux, TOULOUSE
}

INTRACAVERNOUS INJECTION OF PROSTAGLANDIN E1 DURING THE FIRST VISIT : A REPORT OF 414 CASES. The authors report their experience of 414 injections of prostaglandin E1 (prostine). The diagnostic orientation between functional or organic impotence was assessed through questionaires and more particularly on the presence or absence of nocturnal or morning erections. The prostine reconditioning and self injection use techniques are precised : $80 \%$ of positive responses with better results in the oldest patients, whatever their medical antecedents. No relation between positive response to prostaglandin $E !$ and normal nocturnal erections could be found. Patients still presenting erections can present good or bad responses. This confirms the results previously given only allow us to put in evidence the good funtional and anatomic state of cavernous bodies, without any etiological orientation (not mentioning the cases in which these self-injections reveal the presence of deformation, bend or fibrosis). The acceptance of self injection is low (30\%) as well as a regular and prolonged medical observance (only $15 \%$ ). This work confirms the low rate of local complications after repeated injections and the low rate of prolonged erections or priapism during Test 1 . However, these possibilities must incite to prudence as for the generalization of this kind of treatment, out of specialized centers. Key words Prostaglandin El - Erectile impotence. Andrologie, 1991, 1 : 133-135.

Durant ces 9 dernières années, les intra-caverneuses (IC) de produits myo-relaxants (papavérine) associés ou non aux alpha-bloquants (phentolamine), ont créé une véritable révolution dans le traitement de l'impuissance (organique et fonctionnelle).

Toutefois, en dehors de l'intérêt thérapeutique, certains ont vu dans cette technique simple et rapide, un véritable test diagnostique, se basant sur le concept que les réponses négatives aux intra-caverneuses étaient synonymes d'impuissances psychogènes, et les réponses négatives synonymes d'organicité. Un tel raisonnement est erroné et dangereux : 4 à $16 \%$ de complications aigües (priapisme) et autant ou plus de complications secondaires : nodules, fibroses, sont régulièrement observées.

Les travaux d'Hedlund et Anderson (2) sur les effets des prostaglandines sur le tissu érectile humain, puis les travaux d'Hishii et al (3) sur la prostaglandine $\mathrm{El}$ (PGEI) des impuissances, ont ouvert de nouvelles perspectives thérapeutiques. Devant l'absence d'effets secondaires rapportés avec la PGEI intra-caverneuse, et conscients de l'intérêt thérapeutiques des intra-caverneuses dans le traitement des impuissances, nous avons voulu vérifier quel pouvait être l'apport de la PGEI lors de la première consultation. 414 patients ont bénéficié de ces injections de juillet 1989 à juillet 1991. Notre recrutement se fait par l'intermédiaire des Services d'Urologie.

Notre objectif a été quadruple : 1. Étudier les corrélations possibles entre réponse positive à la PGEl et le type d'érections nocturnes cliniquement observées (habituellement mesurées par la N.P.T. ou Nocturnal Penile Tumescence), afin de souligner l'intérêt diagnostique de l'IC pratiquée en première intention ; 2 . vérifier les réponses à la prostaglandine IC lors de pathologies organiques avérées, afin de dégager, outre l'intérêt diagnostique, l'intérêt thérapeutique ; 3 . de vérifier si l'absence d'effets secondaires rapportée antérieurement, était confirmée et enfin 4 . déterminer, à partir de notre étude et de nos impressions, quelles pouvaient être les possibilités du test à la PGE1 pratiqué lors de la première consultation.

\section{MATÉRIEL ET MÉTHODES}

Les 414 patients se répartissent en 6 groupes en fonction de leur âge : groupe $1: 20-30$ ans $(n=14)$, groupes $2: 30-40$ ans $(n=36)$, groupe 3 : 40-50 ans $(n=86)$, groupe $4: 50-60$ ans $(n=135)$, groupe $5: 60-70$ ans $(n=120)$, groupe $6: 70-80$ ans $(n=24)$. Le déroulement de la consultation a été le même pour tous les patients :

- interrogatoire détaillé afin de préciser le type d'impuissance

- recherche systématique de la persistance d'érections nocturnes,
- examen somatique local et général,

- inventaire des pathologies organiques associées et recherche systématique des pathologies méconnues du sommeil (syndrome d'apnée et mouvements périodiques),

- examen döppler avec index de pression pénienne et épreuve d'effort,

- test à la PGE1 en fin de consultation.

Dans 26 cas, soit $6,1 \%$, il s'agissait d'une impuissance primaire et dans 388 cas, d'une impuissance secondaire. Dans ce groupe, 264 patients présentaient une incapacité érectile totale, $(63,37 \%)$, alors qu'elle était relative ou intermittente ou relationnelle dans 124 cas, $(29,9 \%)$. Une éjaculation précoce primaire était retrouvée chez $97,(23,4 \%)$. La qualité des érections nocturnes permettait de différencier 3 groupes étiologiques possibles :

- groupe des érections nocturnes totalement rigides et stables (EN TR $+n=146$ soit $35,3 \%$ ), correspondant au groupe d'impuissances fonctionnelles,

- groupe des érections nocturnes avec simples tumescences ou érections partiellement rigides rendant les rapports impossibles chez 243 patients $(58,6 \%)$.

Une pathologie organique susceptible à elle seule d'expliquer l'impuissance était retrouvée 99 fois, soit dans $23,9 \%$ des cas.

La prostaglandine IC a été reconditionnée pour son utilisation par une dilution dans $11,5 \mathrm{ml}$ de $\mathrm{Na}$ cl à $9 / 1000$, de telle sorte qu'une UI de seringue à insuline corresponde à 1 microgramme de PGEl. Ce conditionnement a été réalisé en milieu aseptique, sous hotte à flux laminaire, permettant le conditionnement de flacons multidoses. La dose utilisée a varié de 5 à 25 microgrammes $(92,6 \%$ des doses se situant entre 15 et 20 microgrammes).

L'injection a été effectuée dans les mêmes conditions, (sujet en décubitus dorsal, mise en place d'un garrot à la base de la verge, injection pratiquée dans le corps caverneux droit, massage en position couchée pendant deux minutes, verticalisation, ablation du garrot, massage vibratoire de 3 à 8 minutes, et jusqu'à une réponse complète, mise en place d'un stimulateur vibratoire 
(Vibrector) dans le cas de réponses insuffisantes. La réponse à l'injection a été cotée comme celle des érections nocturnes : TR $(+)$ : totalement rigide, TR (-) : rigidité incomplète mais suffisante pour la pénétration, et réponse négative (TUMPR) : tumescence partiellement rigide.

\section{RÉSULTATS}

- Une réponse TR (+) a été observée chez 214 patients soit dans $51,69 \%$ des cas.

- Une réponse TR (-) a été notée 128 fois soit dans $30,91 \%$ des cas.

- Une absence de réponse ou une réponse très insuffisante a été constatée chez 72 sujets soit dans $17,39 \%$ des cas (figure 1).

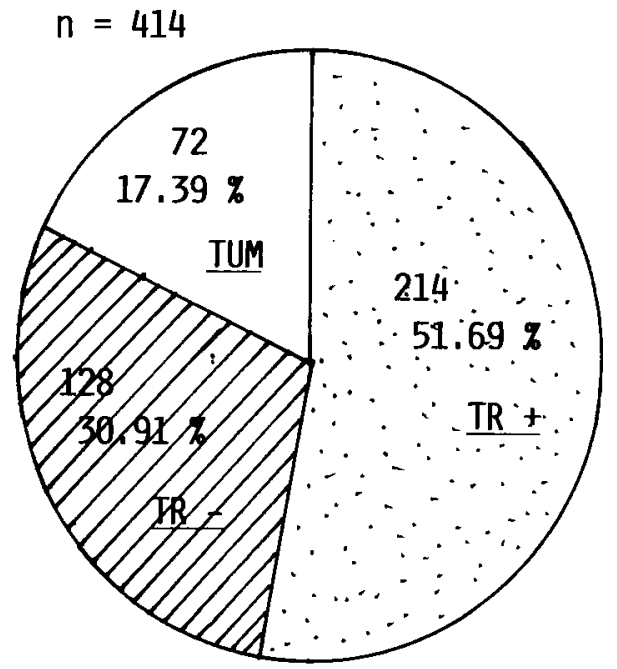

Fig. 1 : Résultats globaux des réponses à la PGE1

La durée de la réponse est variable en fonction des groupes:

- Dans le groupe TR $(+)$ nous avons retrouvé une majorité de réponses durant $1 \mathrm{~h}$ à $3 \mathrm{~h}$ chez 143 patients, soit dans $34,5 \%$ des cas ;

- Les réponses courtes, supérieures à $15^{\prime}$ et inférieures à $1 \mathrm{~h}$ ont été peu nombreuses (47 patients soit $12,6 \%$ des cas)

- Les réponses longues ont été exceptionnelles, 16 patients ayant eu une réponse d'une durée supérieure à $3 \mathrm{~h}$ mais inférieure à $6 \mathrm{~h}$ soit dans $3,8 \%$ des cas, 8 sujets ont présenté une érection prolongée supérieure à $6 \mathrm{~h}$ mais inférieure à $10 \mathrm{~h}$, dont 2 ayant nécessité une ponction aspiration des corps caverneux.

- Dans le groupe TR (-), les réponses sont plus courtes puisque la majorité présente des réponses inférieures à $1 \mathrm{~h}$ mais supérieures à 15' (84 patients soit $20,90 \%$ des cas). Les réponses d'une durée moyenne de 1 à $3 \mathrm{~h}$ ne représentent que 44 patients soit $10,7 \%$ des cas.
Le type de réponse varie selon les groupes d'âge, (fig 2):

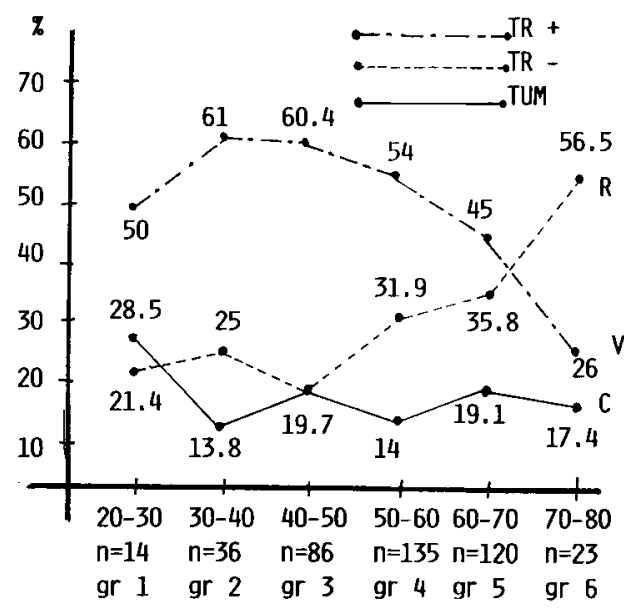

Fig. 2 : Evolution de la réponse à la PGEl en fonction de l'âge

- Le nombre de réponses TR (+) atteint $50 \%$ dans le groupe 1 pour plafonner à $60-61 \%$ dans les groupes 2 et 3 , et chuter à $26 \%$ dans le groupe 6.

- Le nombre de réponses TR (-) subit une progression inverse, partant de $21,4 \%$ dans le groupe 1, pour atteindre $56,5 \%$ dans le groupe 6 .

- Le nombre de réponses négatives (TUM PR) est par contre relativement stable, variant de $13,8 \%$ à $19 \%$ dans les groupes $2,3,4,5$ et 6 , et atteint son maximum dans le groupe des hommes jeunes (groupe 1) dans $28,5 \%$ des cas.

Si l'on cumule les réponses $T R(+)$ et $T R(-)$ permettant un rapport, on s'aperçoit que le nombre de réponses positives dépasse $80 \%$ à $86 \%$ (80 à $86 \%$ ) du groupe 2 au groupe 6 , tandis que le nombre de réponses positives est le plus faible dans le groupe des hommes jeunes de 20-30 ans, ne dépassant pas $71,4 \%$ (figure 3 ).

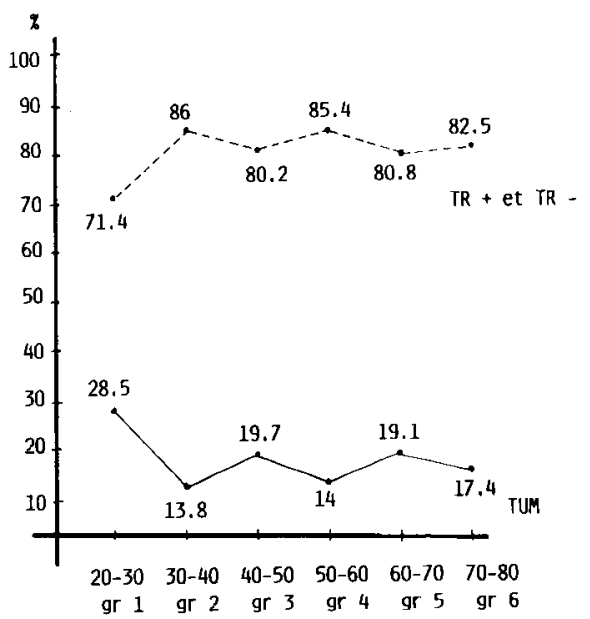

Fig. 3 : Evolution de la réponse à la PGEl en fonction de l'âge. Comparaison entre les groupes TR + et TR - et le groupe TUM.

Réponse à le PGEl en fonction du type d'érections nocturnes : il est résumé dans le tableau 1.
Tableau 1 : Réponse à la PGEl en fonction des érections

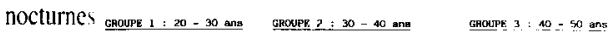

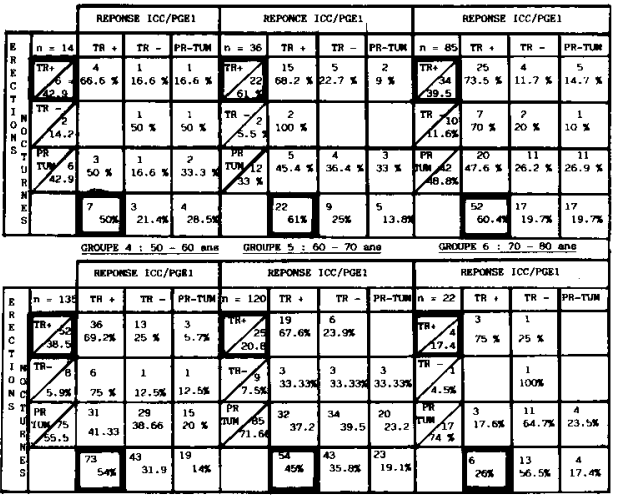

On est surpris de voir qu'il n'y a que $2 / 3$ de sujets EN TR (+) qui répondent très bien à la PGE1, 66 $\%$ dans le groupe 1, avec une progression croissante dans les groupes plus âgés, pour atteindre $75 \%$ dans le groupe 6.

Dans le groupe EN TR (+), le taux de réponses négatives à la PGEl est plus important dans les goupe 1,2 , et $3(16,8 \%, 9 \%$ et $14,7 \%)$, tandis qu'il n'y a pas de réponses négatives dans les groupes plus âgés (5 et 6).

Si l'on considère le groupe EN (-) (PR TUM), on s'aperçoit que la PGEl entraîne des réponses TR (+) dans $50 \%$ du groupe $1,45 \%$ du groupe 2 , $47,6 \%$ du groupe $3,41,3 \%$ du groupe $4,37,2 \%$ du groupe 5 et $17,6 \%$ du groupe 6 .

L'absence totale de réponse à la PGE1 dans le groupe (PR TUM) est plus élevée dans le groupe des patients, groupe $1(33,3 \%)$, tandis qu'elle n'est que de $26 \%$ dans le groupe $3,20 \%$ dans le groupe 4 et $23 \%$ dans les groupes 5 et 6 .

Résultats de la PGEI en fonction des pathologies organiques associées évidentes: Ces pathologies organiques réagissent bien à la PGEl avec réponses TR (+) et TR (-) chez 38 des 48 cas de chirurgie radicale de la prostate ou de la vessie, soit $80 \%$ de bonnes réponses, dans $50 \%$ des cas de chirurgie du carrefour ou d'une artérite, et chez 39 cas de fibrose sur 51.

\section{DISCUSSION}

Nos résultats confirment ceux des précédentes études avec un pourcentage de bonnes réponses TR (+). Toutefois légèrement inférieur à celui d'Ishii (3) $(51,69 \%$ contre $62 \%)$, et inférieur à celui de Sarosdy (6) $(51,69$ contre $66 \%)$. Le pourcentage global de réponses acceptables permettant un rapport sexuel est de l'ordre de $80 \%$ dans notre série (86\% pour Ishii, 3 ).

Nos résultats sont toutefois différents quant à la réponse en fonction de l'âge. En effet, Ishii (3) obtient une réponse d'autant meilleure que le sujet est jeune, et une réponse d'autant moins bonne que le sujet est âgé et qu'il y a une pathologie organique associée. 
Or notre étude montre que le taux de réponses négatives est surtout important dans le groupe 1 , dans lequel les échecs sont en partie liés à des malaises d'origine vagale. Par contre, chez le sujet âgé, il n'y a pas de diminution de la réponse. La réponse permet la reprise d'une sexualité, et ceci quels que soient les facteurs organiques sous-jacents, ce qui va à l'encontre des précédentes publications sur l'intérêt diagnostique de la PGE1.

Une rêponse positive indique donc l'intégrité fonctionnelle et anatomique des corps caverneux avec comme corollaire la possibilité d'utiliser l'injection de prostaglandine comme prothèse chimique avec $80 \%$ de bons résultats dans les artériopathies, avec ou non pathologie associée du carrefour. Une réponse négative n'a pas de signification puisqu'il y a 12 sujets sur 72 qui ne répondent pas à la PGE1, mais qui présentent une réponse tout à fait normale avec l'association Papavérine-Phentolamine.

Ainsi, l'intérêt de la prostaglandine El injectée en intracaverneuse est essentiellement thérapeutique. Toutefois, l'acceptabilité de l'idée des autoinjections n'est que de $14 \%$ dans le groupe 1 (2/14), $9 \%$ dans le groupe $2(3 / 36)$, et se situe aux alentours de 30 à $45 \%$ dans les autres groupes $(33 \%$ dans le groupe $3,35 \%$ dans le groupe $4,45 \%$ dans le groupe $5,31 \%$ dans le groupe 6). L'utilisation réelle des auto-injections en tant que traitement, est faible (seulement 61 patients utilisent les auto-injections depuis maintenant un à deux ans, soit $15 \%$ des cas).

L'apparition de complication aigües lors de la première injection est exceptionnelle : deux ponctions à la 10 ème heure, soit $0,4 \%$ ayant seulement été nécessaires. L'apparition de complications à long terme est également exceptionnelle. Un cas de nodule avec déformation, mais réponse toujours positive chez un diabétique, et un cas d'accentuation d'une coudure chez un autre sujet, avec persistance d'une bonne réponse également.

Une amélioration de la réponse au fur et à mesure des injections est souvent rapportée, mais non quantifiée dans notre travail, avec des doses initiales de 15 microgrammes, qui seront réduites dans certains cas à 3 ou 4 microgrammes. Un effet bénéfique sur l'éjaculation est également noté, en raison du caractère un peu douloureux de celle-ci ; mais la douleur fréquemment notée chez Sarosdy (33\% des cas) n'a été une source d'abandon que dans $5 \%$ de notre série.

\section{CONCLUSION}

Notre étude confirme celle des précédents auteurs, en ce sens que la PGEl en intra-caverneux est suivie d'une réponse. TR (+) et TR (-) dans plus de $80 \%$ des cas, et cette réponse est d'autant meilleure que nous arrivons dans les tranches d'âge plus élevées, où la pathologie organique est plus fréquente.

L'idée que la PGEl pouvait être utile sur le plan du diagnostic étiologique semble infirmée, étant donné qu'il n'y a pas de relation entre les réponses positives à la prostaglandine et la qualité des érections nocturnes. L'avantage de la PGEl par rapport aux autres substances (Papavérine, Phentolamine) réside avant tout dans sa facilité et sa simplicité d'utilisation, ce qui est confirmé par le faible pourcentage d'érections prolongées nécessitant une ponction, et le faible pourcentage de complications secondaires de type nodules ou fibrose.

Le seul véritable inconvénient de la prostaglandine est sa difficulté de conditionnement et de conservation.

\section{RÉFÉRENCES}

1 - Calvet U.,Corraze J. : Intérêt de la polysomnographie "lourde" couplée à la pléthysmographie pénienne nocturne. Sexologies, 19911 : 13-18 juillet.

2 - Hediund S, Anderson K. E. : Contraction and relaxation induced by some prostanoïds in isolated penile erectile tissue and cavernous artery, J. Urol., $1985,134: 1245$.

3 - Ishii N., Watanabe H., Irisawa C., Kikuchi Y., Kubota Y., Kawamura S., Suzuki K., Chiba R., Toriwa M., Shirai M. : Intracavernous injection of prostaglandin $\mathrm{E} 1$ for the treatment of erectile impotence. J. Urol., 1988, 141 : 323-325:

4 - Lee L.M., Stevenson R.W.D., Szaz G. : Prostaglandin E1 versus phentolamine / papaverine for the treatment of erectile impotence : a double blind comparaison. J. Urol., 1989, $141:$ 549-551.

5 - Reiss I.K. : Use of prostaglandin E1 for papaverine. Failed erections Urology 1989, 33, 1 : 15-16.

6 - Sarosdy M.F., Hudnall C.H., Erikson D.R., Hardin TH. C., Novicki D.E. : A prospective double blind trial of intracorporal papaverine versus prostaglandin E1 in the treatment of impotence. J. Urol., 1988, 141: $551-553$.

RÉSUMÉ : Les auteurs rapportent 414 cas d'utilisation de la prostaglandine $\mathrm{E} 1$ en première intention. La dose utilisće dans la majorité des cas a été de 15 microgrammes, avec des réponses positives dans près de 80 \% des cas. L'acceptabilité et la régularité dans la pratique des auto-injections est faible ( 30 et $15 \%$ ). L'intérêt de la prostaglandine $\mathrm{E} 1$ réside dans la rareté des complications, qu'il s'agisse de nodules, de déformations et surtout de priapisme. Motsclés : Prostaglandine E1, Impuissance erectile. Andrologie, 1991, : 133-135 